\title{
Médiévales
}

Langues, Textes, Histoire

73 | automne 2017

Le texte à l'épreuve du numérique

\section{L'édition numérique du Livre de jostice et de plet (Élec), point de départ de la constitution d'une base prosopographique (Omeka)}

The Scholarly Digital Edition of the Livre de jostice et de plet (Élec) : A

Starting Point for Creating a Prosopographical Database with Omeka CMS

\section{Graziella Pastore}

\section{OpenEdition}

Journals

Édition électronique

URL : https://journals.openedition.org/medievales/8173

DOI : 10.4000/medievales. 8173

ISSN : $1777-5892$

\section{Éditeur}

Presses universitaires de Vincennes

\section{Édition imprimée}

Date de publication : 15 décembre 2017

Pagination : $31-45$

ISBN : 978-2-84292-801-8

ISSN : 0751-2708

\section{Référence électronique}

Graziella Pastore, « L'édition numérique du Livre de jostice et de plet (Élec), point de départ de la constitution d'une base prosopographique (Omeka) », Médiévales [En ligne], 73 | automne 2017, mis en ligne le 28 février 2019, consulté le 23 avril 2022. URL : http://journals.openedition.org/medievales/ 8173 ; DOI : https://doi.org/10.4000/medievales.8173 


\section{L'édition numérique du Livre de jostice et de plet (Élec), point de départ de la constitution d'une base prosopographique (Omeka)}

L'augmentation des données numériques dans les sciences humaines a introduit de nouveaux défis pour simplifier l'accès, la gestion et l'interopérabilité des données, notamment prosopographiques. Les fichiers d'autorité étant aujourd'hui toujours plus nombreux et complexes (autorités BnF, BPN Biografisch Portaal van Nederland, GND Gemeinsame Normdatei, IdRef, ISNI, LC NAF, VIAF, etc.), des outils émergent pour améliorer le dialogue entre les différentes sources, d'une part, et les nombreux utilisateurs et producteurs de données (les chercheurs, les professionnels de l'information, les institutions, etc.), d'autre part ${ }^{1}$. Si l'intérêt pour le réseau et le contexte de production des données ainsi que l'attention à leur format s'avèrent indispensables dans les phases de planification d'une base prosopographique, il reste souvent difficile au sein d'une équipe de recherche de trouver le bon équilibre entre les objectifs scientifiques, les préconisations descriptives et les compétences techniques et numériques nécessaires pour mener à bien un tel projet dans un temps défini et à des coûts raisonnables. L'interopérabilité et la pérennité d'une base prosopographique sont affectées directement par ces contraintes de faisabilité.

Cette contribution constitue une étude de cas portant sur une tentative concrète de relier, d'un côté, un projet d'édition numérique d'un texte médiéval, le Livre de jostice et de plet, de l'autre, un projet de base prosopographique des personnes citées dans ce texte ${ }^{2}$.

1. Voir par exemple les projets RAMP (Remixing Archival Metadata Project) $<$ https:// tools.wmflabs.org/ramp/> ; ICA AtoM (Access to Memory) <https://www.accesstomemory. org/fr/> ; SNAC (The Social Networks and Archival Context Projet) <http://socialarchive. iath.virginia.edu/>; symogih.org, plateforme collaborative de gestion de l'information historique <http://www.symogih.org/>.

2. Cette contribution porte sur deux volets de nos recherches menées à l'École nationale des chartes entre septembre 2013 et octobre 2016. Nous nous sommes occupée de l'étude 
L'objectif de ce projet, mené au sein de l'équipe PARTHENOS ${ }^{3}$, était tout d'abord de préconiser un système de publication et de gestion des fichiers d'autorité à la portée de chercheurs n'ayant que peu de compétences informatiques et souhaitant créer une base prosopographique en utilisant des schémas de description standard (EAD, EAC-CPF, METS, TEI, RDF, etc.), dans le cadre d'un projet personnel ou d'équipe. Nous avons tenté de modéliser un cas de figure (use case) suffisamment simple à mettre en place et à reproduire, pourvu d'une chaîne d'édition bien documentée, de manière à pouvoir répondre aux besoins des différentes communautés scientifiques, plus particulièrement dans le cadre de descriptions d'archives et de projets de recherche en histoire, mais suffisamment souple pour s'adapter à d'autres domaines ${ }^{4}$.

Suite à une première phase d'exploration d'autres projets en cours et des solutions existantes, nous avons mieux précisé l'orientation de la solution de notre cas d'étude du point de vue du format, de l'outil de visualisation et de l'ensemble des données à inclure dans la base. EAC-CPF, un schéma de métadonnées basé sur le langage XML et permettant de structurer les descriptions des collectivités, des personnes ou des familles en respectant les indications de la deuxième édition de la norme ISAAR $(\mathrm{CPF})^{5}$, s'est imposé comme format de départ de la réflexion en raison de sa diffusion ainsi que par la complexité de sa structure, qui permet de détailler finement

et de l'édition de la partie inédite du Livre de jostice et de plet au sein du Centre Jean Mabillon grâce au financement de la Ville de Paris («Research in Paris » 2014), du Labex Hastec (contrat postdoctoral, 2015) et de l'Université Franco-Italienne (« Vinci » 2016). Le développement de la base prosopographique Omeka a été conçu en 2016 pendant un stage à l'Inria (Paris) dans le cadre des activités du master « Technologies numériques appliquées à l'histoire » (Énc).

3. PARTHENOS (Pooling Activities, Resources and Tools for Heritage E-research Networking, Optimization and Synergies) est un projet financé dans le cadre du programme européen Horizon 2020, composé par un consortium de quinze partenaires européens. Ses objectifs portent en particulier sur la définition de normes communes et l'utilisation de formats standards pour les données de la recherche dans les domaines des SHS et du patrimoine culturel. Nous avons collaboré au Work Package 4 (WP4), porté par l'Inria (Paris) et coordonné par Laurent Romary. Pour des précisions sur ce projet, voir <http://www. parthenos-project.eu/>.

4. L'ensemble de ce projet est expliqué en détail dans notre mémoire pour le diplôme de master « Technologies numériques appliquées à l'histoire », "Création et gestion d'une base de données prosopographiques EAC-CPF avec le CMS Omeka. Les officiers et jurisconsultes cités dans Li livres de jostice et de plet (Paris, BnF, fr. 2844), <http://josticeetplet.humanum.fr>, École nationale des chartes, 2016. L'essentiel des informations techniques figure également dans l'entrepôt GitHub du projet PARTHENOS <https:/github.com/ ParthenosWP4/standardsLibrary/tree/master/prosopography> ainsi que dans notre entrepôt personnel <https://github.com/sgraziella>.

5. Ce schéma est maintenu par la Society of American Archivists en partenariat avec la Bibliothèque nationale de Berlin <http://eac.staatsbibliothek-berlin.de/> ; voir aussi la description sur le site de la $\mathrm{BnF}<\mathrm{http}$ ///www.bnf.fr/fr/professionnels/formats_catalogage/ a.f_eac.html>. 
la description des personnes; le workflow construit autour de ce schéma peut donc être aisément adapté à d'autres systèmes d'encodage plus généralistes qui intègrent la description des personnes dans leurs schémas de données (comme c'est le cas par exemple de l'élément <person> pour la TEI - Text encoding initiative $)^{6}$. Pour l'outil de visualisation, un consensus s'est vite établi autour de Omeka, un système de gestion de contenus (CMS - Content Mangement System) offrant un terrain d'exploration intéressant ; ce système, particulièrement apprécié pour la mise en ligne de bibliothèques numériques et suivi par une active communauté de développeurs ainsi que par la TGIR Huma-Num ${ }^{7}$, permet aisément la création d'un site web et la gestion de plusieurs profils d'utilisateurs ${ }^{8}$. Enfin, quant aux données à inclure dans la base, nous avons choisi un corpus avec lequel nous étions familier : le petit corpus des personnes citées dans le Livre de jostice et de plet, une compilation juridique $\mathrm{du} \mathrm{XIII}^{\mathrm{e}}$ siècle, que nous présenterons plus bas ; il se compose d'une quinzaine de personnes qui ont donné lieu à des recherches biographiques, principalement dans le but d'identifier le compilateur de cet ouvrage et de connaître son milieu.

La solution décrite ici est donc le résultat d'une combinaison entre la recherche et l'expérience pratique : un outil de gestion de fichiers d'autorités avec des fonctionnalités d'édition permettant de publier les données en ligne sans une courbe d'apprentissage abrupte.

\section{Le Livre de jostice et de plet}

Le texte

Le Livre de jostice et de plet est une compilation juridique rédigée en français, vraisemblablement dans la région d'Orléans, dont la composition se situe sans doute après 1259 , durant le règne de Louis IX $^{9}$. Ce texte est

6. Pour la description de cet élément, voir <http://www.tei-c.org/release/doc/tei-p5-doc/ $\mathrm{fr} / \mathrm{html} /$ ref-person.html>.

7. Pour Omeka, voir <http://omeka.org/>. Omeka est un logiciel libre (licence GPL), développé avec les technologies PHP et MySQL, qui gère nativement des métadonnées au format Dublin Core échangeables via OAI-PMH. Pour le consortium Huma-Num, voir $<$ http://www.huma-num.fr/> et <https://www.nakalona.fr/>.

8. Outre les projets internationaux signalés sur le site Omeka $<$ http://omeka.org/codex/ Sites_Using_Omeka>, voir par exemple la Bibliothèque patrimoniale numérique de l'École des mines <https://patrimoine.mines-paristech.fr> et les quatre bibliothèques numériques de l'Université de Poitiers, dont notamment celle consacrée aux coutumiers du Centre-Ouest $<\mathrm{http}$ ///coutumiers .edel.univ-poitiers.fr/>.

9. Le titre de l'ouvrage est tiré de la table des matières : « Ci commance li livres de jostice et de plet », f. 199c. Le texte contient des éléments de datation post quem : voir Li livres de jostice et de plet, publié pour la première fois d'après le manuscrit unique de 
conservé par un seul manuscrit (Paris, BnF, fr. 2844) de deux cents feuillets en parchemin de grand format $(350 \times 270 \mathrm{~mm}$; justification : $235 \times 163$ $\mathrm{mm})$; sa réalisation semble, d'après l'écriture et le décor, de peu postérieure à la rédaction de l'œuvre ${ }^{10}$.

Outre les sections consacrées au droit coutumier, le Livre de jostice et de plet rassemble de nombreux extraits tirés du droit dit « savant », c'està-dire du droit romain et du droit canon, et tout particulièrement du Digeste, partie centrale du Corpus juris civilis de Justinien (deux cents paragraphes sur les trois cent quarante-deux qui composent l'ouvrage). Aussi, le plan de cette compilation, organisé en vingt livres, suit de près celui du Digeste, dont il reprend également la tripartition médiévale (Digeste vieux [Digestum vetus], Infortiat [Digestum infortiatum], Digeste neuf [Digestum novum]).

La production de ce texte s'inscrit dans une époque et dans un milieu particulièrement favorables à l'émergence des études juridiques, qui conduira Orléans à être l'un des lieux les plus réputés pour l'enseignement du droit romain en France. Le compilateur du Livre de jostice a joué un rôle actif dans la rédaction du texte : il ne s'est pas limité à la sélection des sources mais il est intervenu avec des ajouts et des modifications substantielles, quoique distribués de manière inégale sur l'ensemble de l'ouvrage. Ses interventions, concentrées principalement sur les dix premiers livres (c'està-dire dans les sections contenant les extraits du Digeste vieux et, dans une moindre mesure, de l'Infortiat) consistent principalement à retravailler les sentences du Digeste, notamment en les attribuant occasionnellement non pas aux jurisconsultes romains, mais à des baillis, à des officiers et à des conseillers actifs sous le règne de Louis IX (fig. 1). Au-delà des questions que ce type d'intervention pose du point de vue de l'acclimatation du droit romain à la coutume et des rapports entre les personnes évoquées par le compilateur et les idées exprimées dans le texte latin du Digeste, ce réaménagement des sources savantes montre bien l'ancrage territorial et politique du texte, principalement dans l'Orléanais, mais aussi dans une aire géographique plus vaste qui touche le Maine et la basse Normandie. Ce sont donc ces personnes, que nous évoquerons plus tard, qui composent le corpus de la base prosopographique consacrée au Livre de jostice et de plet.

la Bibliothèque nationale par [P.N.] Rapetti, avec un glossaire des mots hors d'usage, éd. P. CHABAILle, Paris, 1850 (Collection de documents inédits sur l'histoire de France. Première série, Histoire Politique), p. 170, 225 et 304. Pour une introduction à l'œuvre, voir les notices de J. Foviaux (Dictionnaire des lettres françaises. Moyen Âge, Paris, 1992, p. 955-958) et de G. Giordanengo (P. ARABEYRE, J.-L. HALPÉRIN et J. KRYNEN éd., Dictionnaire historique des juristes français $X^{e}{ }^{e}-X X^{e}$ siècle, Paris, 2015, p. 669-670).

10. Pour la description du manuscrit et pour l'étude philologique, voir G. PASTORE et F. DUVAL, «La tradition française de l'Infortiat et le Livre de jostice et de plet », Bibliothèque de l'École des chartes, 171 (2013), p. 199-225. 


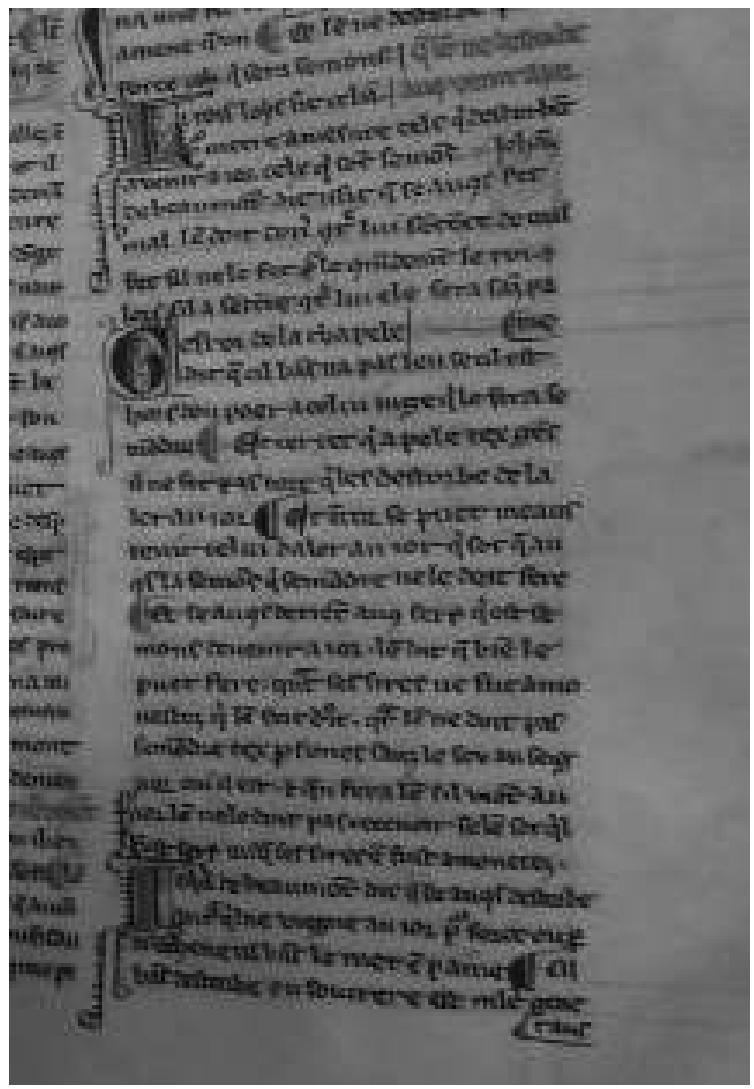

1. Livre de jostice et de plet (Paris, BnF, ms. fr. 2844, f. 26b.)

Pour l'édition de ce passage, voir <http://elec.enc.sorbonne.fr/josticeetplet/livre2/art_09>.

\section{L'édition dans la collection Élec}

Jusqu'à présent, le Livre de jostice n'était connu qu'à travers une édition partielle, réalisée en 1850 par Pierre-Nicolas Rapetti (1811-1885), docteur en droit et ancien professeur au Collège de France. Son édition ne livre qu'à peine une moitié de l'ouvrage : les extraits traduits d'après le Digeste, constituant la partie la plus importante du Livre de jostice, étaient restés complètement inédits et n'avaient fait l'objet que de quelques sondages lexicographiques ${ }^{11}$. L'édition numérique complète, disponible

11. L'intérêt d'une édition complète ainsi que d'une révision de l'édition Rapetti ont été signalés entre autres par J. SEPPÄNEN, « "Livre de jostice et de plet" - Un texte à rééditer ? ", Neuphilologische Mitteilungen, 91/2 (1990), p. 153-155. 
dans la collection Élec (Éditions en ligne de l'École des chartes) depuis novembre 2016, permet enfin de connaître le Livre de jostice dans son intégralité ${ }^{12}$. Le texte édité par Rapetti, ainsi que la structure formelle de son édition - qui annonçait aussi les titres des sections qu'il n'avait pas choisi d'éditer - a servi de base à la création de la version numérique. Plus que d'édition complète, on pourra alors parler d'édition " complétée ».

Chacun des vingt livres qui composent le Livre de jostice est ainsi téléchargeable au format XML, avec un balisage TEI a minima qui ne rend compte que de la structure de l'ouvrage (division en titres, chapitres, paragraphes et interventions des éditeurs). Les sections nouvellementéditées, qui s'affichent en bleu dans le site, ne présentent qu'un apparat critique réduit, faisant seulement état des corrections apportées au manuscrit; le glossaire et des notes explicatives plus détaillées seront réservés à l'édition papier (fig. 2).

\section{La constitution de la base prosopographique Omeka}

\section{Le corpus}

La première étape de constitution de la base a été la création des notices prosopographiques des personnes citées dans le Livre de jostice selon le schéma EAC-CPF/XML. À l'exception de Blanche de Castille et de Louis IX, la plupart des personnes mentionnées dans le Livre de jostice ne disposent pas de notices d'autorité d'après les référentiels (Autorités $\mathrm{BnF}$, VIAF, etc.). Le corpus a donc été créé ex novo, principalement en s'appuyant sur les informations contenues dans une contribution publiée par Henri Stein en $1918^{13}$. Les recherches biographiques de H. Stein se sont concentrées plus particulièrement sur les personnes suivantes, dont les notices constituent le corpus de notre base : Étienne, comte de Sancerre, petit-fils de Thibaud IV comte de Champagne, seigneur de Saint-Brisson près de Gien et de Châtillon-sur-Loing, qui exerça la fonction de bouteiller de France ; Jean de Beaumont, grand chambellan dès 1232, conseiller de Louis IX, nommé chambrier de France en 1241 ; Adam, peut-être à identifier avec Adam de Beaumont, Adam de Villebon ou Adam de Milly ; Geoffroy de la Chapelle, panetier de France et conseiller de Louis IX ; Jean le Monoyer, bailli d'Orléans en 1248-1253, châtelain de Sens au moins de 1263 à 1274 ; Renaud de Tricot, bailli de Gisors en 1235-1237 ; maître Simon, peut-être à identifier avec magister Simon de Poigniis, clerc du roi

12. Voir <http://elec.enc.sorbonne.fr/josticeetplet/>. L'édition est gérée à travers le système de gestion Diple proposant une méthode et des modules pour faciliter la publication en ligne de corpus XML, voir <http://elec.enc sorbonne.fr/diple/theme/enc/>.

13. H. STEIN, «Conjectures sur l'auteur du Livre de jostice et de plet », extrait de la Nouvelle revue historique de droit français et étranger, 1918. 

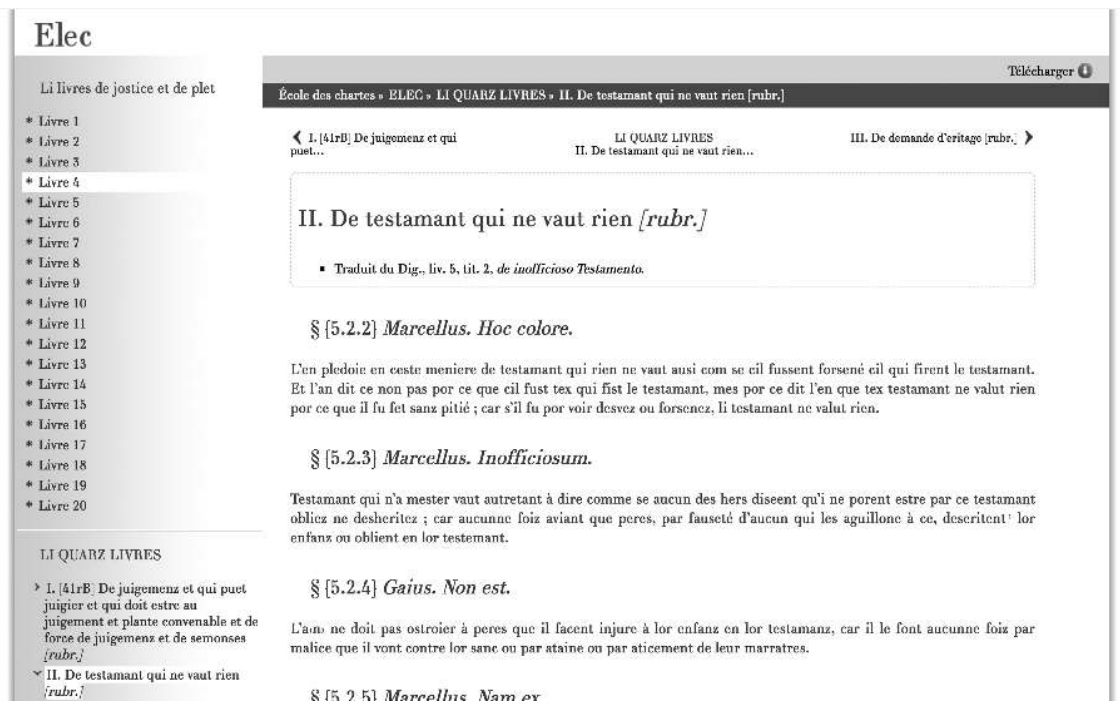

2. L'édition dans la collection Élec

\section{Sources imprimées}

(Stein 1918, etc.)

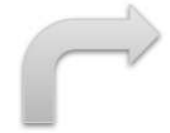

Output EAC-CPF/XML (plugin ExportEacCpf)

Création de la notice Omeka (mapping Dublin Core + Item Type Metadata)

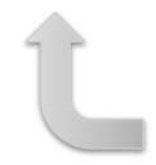

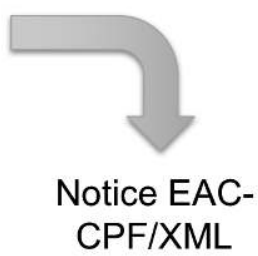
CPF/XML

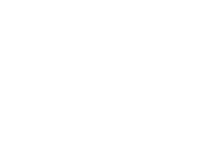

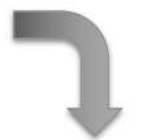

Input

1ère transformation XSLT :

EAC-CPF to XML

(extraction des données)

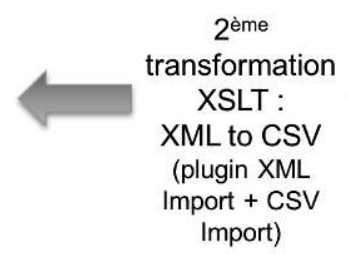

3. Le flux des données 
en 1260 ; monseigneur Jean de Saint-Clair, dont la famille serait originaire de Saint-Clair-sur-Epte ; monseigneur Guillaume de Neuilly, qui tirait son nom de la localité de Neuilly-en-Thelle, dans le comté de Clermont-enBeauvaisis ; enfin, la dame de Chevry, probablement originaire du Gâtinais.

Les sources évoquées par H. Stein étant pour la plupart disponibles dans Gallica, nous avons pu vérifier directement les informations sur les textes, sauvegarder les liens vers les ressources numériques à travers Zotero pour ensuite pouvoir les intégrer aux notices de la base et créer un onglet consacré à la bibliographie. Une partie des notices produites est disponible en ligne dans un entrepôt GitHub ${ }^{14}$.

\section{Le flux des données}

Suite à la création du corpus, le flux des données dans le CMS Omeka, schématisé par la figure 3, comporte trois étapes principales, dans l'ordre suivant (fig. 3) :

1. importation de la notice EAC-CPF au format CSV à travers une transformation XSL (en deux étapes) ;

2. alignement des métadonnées à travers l'interface Omeka (mapping);

3. récupération des données grâce à la sortie EAC-CPF.

Nous avons travaillé avec le CMS Omeka (versions 2.3 et 2.4) et installé les extensions suivantes : Catalog Search (version 1.0.3 par Lincoln A. Mullen) ${ }^{15}$, CSV Import+ (version 2.2 perfectionnée par Daniel Berthereau) ${ }^{16}$; Dublin Core Extended (version 2.0.1, Roy Rosenzweig Center for History and New Media) ${ }^{17}$; XML Import (version 2.15, Daniel Berthereau) ${ }^{18}$; ExportEacCpf (version 0.1, Graziella Pastore et Luca Foppiano ${ }^{19}$, créée à l'occasion de ce projet) ; Simple Pages (version 3.0.5 par Roy Rosenzweig Center for History and New Media) ${ }^{20}$; Zotero Import (version 2.0.2 par Roy Rosenzweig Center for History and New Media) ${ }^{21}$. Dans le cadre de la mise en ligne à travers Huma-Num ${ }^{22}$, nous avons aussi intégré les extensions NAKALA import (version 3.0) et NAKALA export (version 1.0.0), développées par Limonade \& Co-Franck Dupont pour Huma-Num.

14. Voir <https://github.com/sgraziella/prosopography_LJP>.

15. <https://github.com/lmullen/plugin-CatalogSearch>.

16. <https://github.com/Daniel-KM/CsvImportPlus>.

17. <https://omeka.org/add-ons/plugins/dublin-core-extended/>.

18. <https://github.com/Daniel-KM/XmlImport>. L'extension ArchiveFolder souhaite substituer les extensions CSV Import Plus et XML Import, voir <https://github.com/Daniel$\mathrm{KM} /$ ArchiveFolder $>$.

19. <https://github.com/sgraziella/ExportEacCpf>.

20. <http://omeka.org/codex/Plugins/SimplePages_2.0>.

21. <http://omeka.org/codex/Plugins/ZoteroImport_2.0>.

22. Voir infra. 


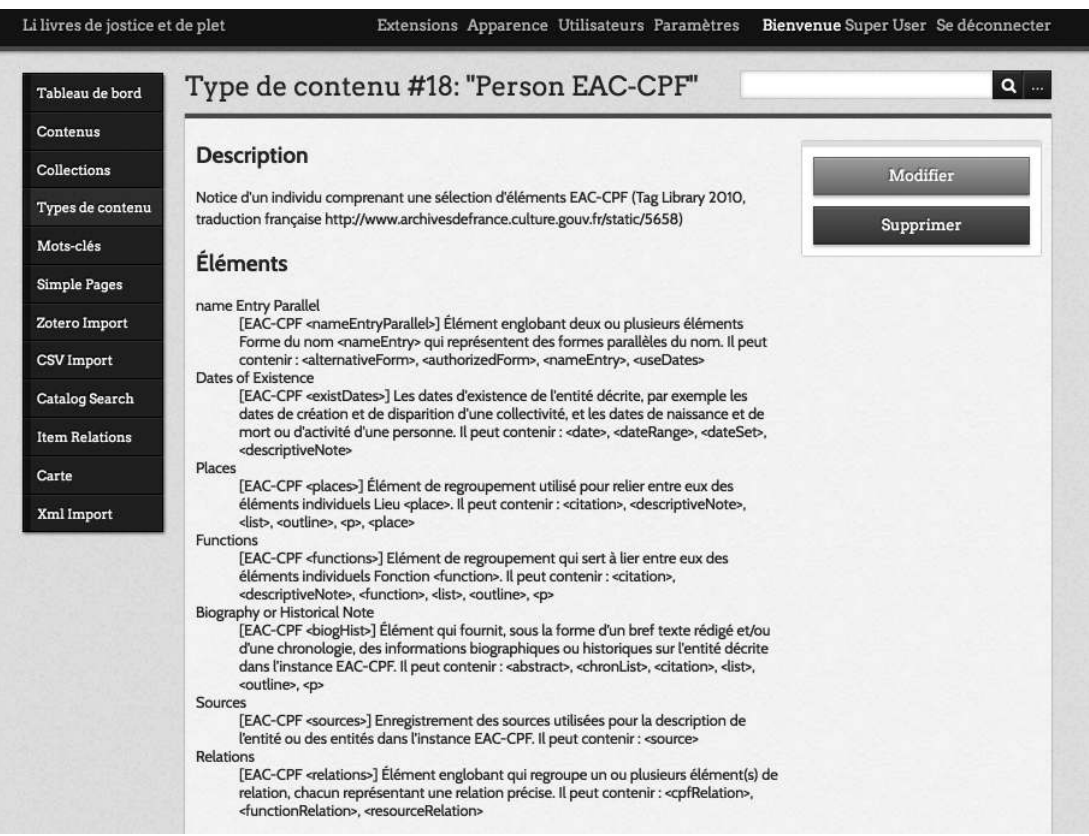

Source : Interface d'administration du CMS Omeka.

\section{Le type de contenu Person EAC-CPF}

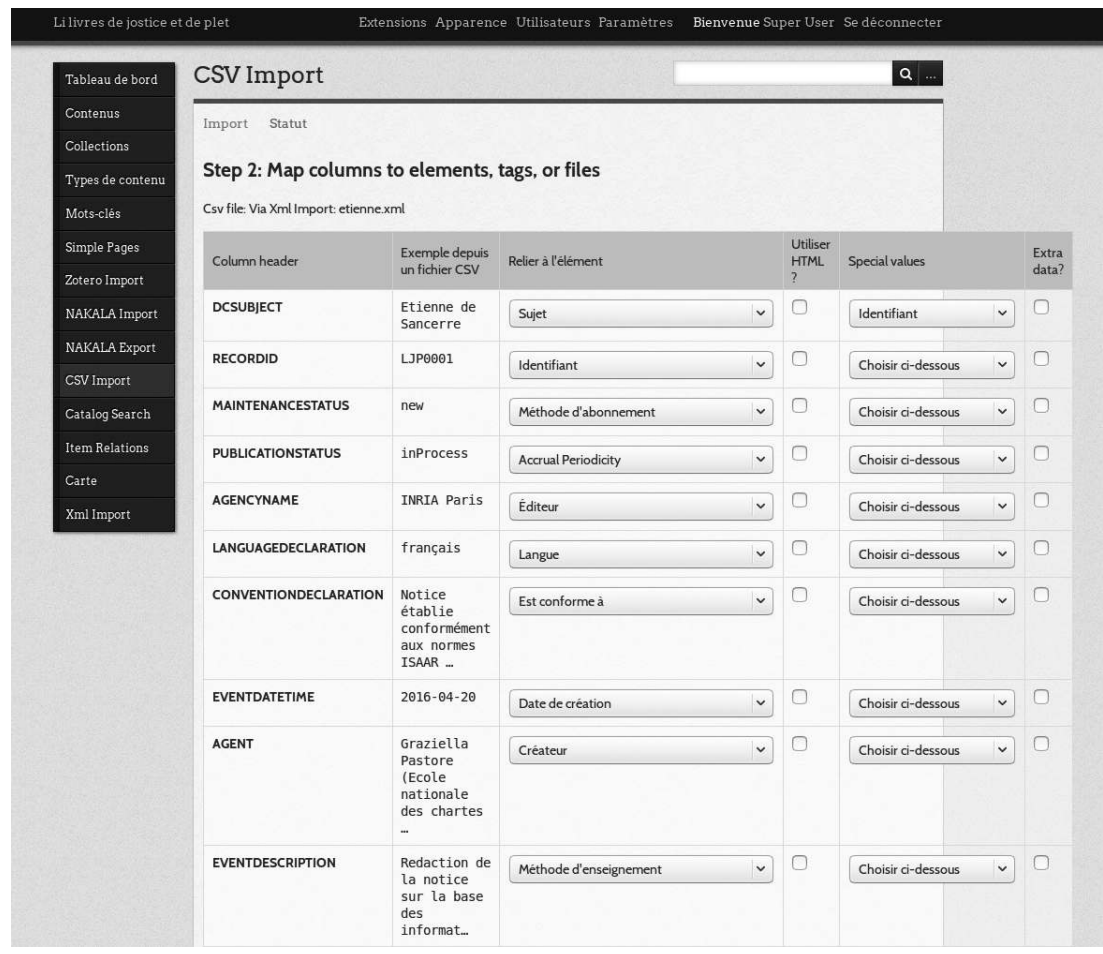

Source : Interface d'administration du CMS Omeka.

5. Le mapping des métadonnées depuis l'interface Omeka 
L'importation des notices

Omeka permet de décrire des types particuliers de contenu, en choisissant des types par défaut (Text, Moving Image, Oral History, Sound, Person, etc.), ou en ajoutant un nouveau type de contenu (item type) depuis l'interface d'administration. À travers des extensions dédiées, Omeka permet aussi d'ajouter, au jeu de métadonnées Dublin Core, d'autres schémas (element set) pouvant être associés à tous les contenus ${ }^{23}$. Si des solutions existent pour l'importation des contenus XML, aucun ensemble d'éléments n'est actuellement disponible à notre connaissance pour gérer les contenus EAC-CPF/XML. En l'absence d'une extension spécifique, nous avons choisi de nous servir de l'extension XML Import citée plus haut, en appliquant à nos fiches EAC-CPF une transformation XSLT en amont afin de permettre ensuite de récupérer les données au format $\mathrm{CSV}^{24}$. En même temps, nous avons créé un nouveau type de contenu, Person EAC$C P F$, qui intègre au type Person fourni par défaut par Omeka (contenant : Birth Date, Birthplace, Death Date, Occupation, Biographical Text, Bibliography) un choix d'éléments EAC-CPF : <NameEntryParallel $>$, $<$ existDates $>,<$ places $>,<$ functions $>,<$ biogHist $>,<$ sources $>,<$ relations $>25$. On remarquera qu'il s'agit d'éléments englobants, dont la structure interne devra être rétablie dans la phase d'exportation des données.

Dans la figure 4 nous indiquons les nouveaux éléments «Person EAC-CPF Item Type Metadata » que nous avons créés en fonction du projet.

L'alignement des métadonnées

Dans le tableau ci-dessous, nous indiquons les correspondances entre les éléments EAC-CPF que nous avons mis en valeur et les éléments Dublin Core $^{26}$. Cet alignement est à réaliser manuellement dans le système Omeka depuis l'interface de l'extension CSV Import (fig. 5).

23. Voir la procédure à l'adresse : <https://omeka.org/codex/Creating_an_Element_ Set> ; pour d'autres détails, voir <http://omeka.org/forums-legacy/topic/element-setexample>.

24. <https://github.com/sgraziella/prosopography_LJP/blob/master/EACtoXML/ EACtoXML_DC.xsl>.

25. La description détaillée des éléments est disponible dans le fichier Readme de l'extension ExportEacCpf<https://github.com/sgraziella/ExportEacCpf>.

26. Nous signalons que les champs DCSUBJECT et NAMEENTRY_TITLE correspondent tous les deux au contenu de l'élément <nameEntry > des notices EAC-CPF; le premier intervient pour permettre le bon fonctionnement de l'extension Catalog Search, alors que le deuxième est utilisé pour créer le titre du contenu (item) Omeka. 
Alignement Dublin Core (mapping)

\begin{tabular}{|c|c|c|c|}
\hline $\begin{array}{l}\text { Éléments Omeka/ } \\
\text { EAC-CPF }\end{array}$ & Exemple & Dublin Core (EN/FR) & Special values \\
\hline DCSUBJECT & Renaud de Tricot & Subject/Sujet & \\
\hline RECORDID & LJP006 & Identifier/Identifiant & $\begin{array}{l}\text { Identifier/ } \\
\text { Identifiant }\end{array}$ \\
\hline $\begin{array}{l}\text { MAINTENANCE } \\
\text { STATUS }\end{array}$ & new & $\begin{array}{l}\text { Accrual method/Méthode } \\
\text { d'abonnement }\end{array}$ & \\
\hline $\begin{array}{l}\text { PUBLICATION } \\
\text { STATUS }\end{array}$ & inProcess & $\begin{array}{l}\text { Accrual policy/Politique } \\
\text { d'acquisition }\end{array}$ & \\
\hline AGENCYNAME & INRIA Paris & Publisher/Editeur & \\
\hline $\begin{array}{l}\text { LANGUAGE } \\
\text { DECLARATION }\end{array}$ & français & Language/Langue & \\
\hline $\begin{array}{l}\text { CONVENTIONDE- } \\
\text { CLARATION }\end{array}$ & $\begin{array}{l}\text { Notice établie confor- } \\
\text { mément aux normes } \\
\text { ISAAR... }\end{array}$ & $\begin{array}{l}\text { Conforms To/Est conforme } \\
\text { à }\end{array}$ & \\
\hline EVENTDATETIME & 2016-05-31 & $\begin{array}{l}\text { Date Created/Date de } \\
\text { création }\end{array}$ & \\
\hline AGENT & Graziella Pastore & Creator/Créateur & \\
\hline $\begin{array}{l}\text { EVENT } \\
\text { DESCRIPTION }\end{array}$ & $\begin{array}{l}\text { Rédaction de la } \\
\text { notice sur la base des } \\
\text { informations... }\end{array}$ & $\begin{array}{l}\text { Instructional Method/ } \\
\text { Méthode d'enseignement }\end{array}$ & \\
\hline $\begin{array}{l}\text { DESCRIPTIVE } \\
\text { NOTE }\end{array}$ & $\begin{array}{l}\text { Figure dans l'édition } \\
\text { de « Li livres de } \\
\text { jostice et de plet » } \\
\text { aux pages... }\end{array}$ & $\begin{array}{l}\text { Bibliographic Citation/ } \\
\text { Référence bibliographique }\end{array}$ & \\
\hline ENTITYTYPE & personne & Item Type/Type & \\
\hline $\begin{array}{l}\text { NAMEENTRY } \\
\text { TITLE }\end{array}$ & Renaud de Tricot & Title/Titre & \\
\hline
\end{tabular}

Suite à l'étape d'importation, les notices EAC-CPF font désormais partie des contenus Omeka : chaque notice peut être éditée directement à travers l'interface de gestion du CMS ; elle dispose également d'un lien vers le fichier XML d'origine.

\section{La récupération des données}

Grâce à ses fonctions de base et à travers des extensions spécifiques, Omeka permet d'exporter les contenus sous plusieurs formats (ATOM, DC-RDF, Omeka-XML, METS, etc.). Étant donné qu'il n'existe aucune extension dédiée à l'exportation au format EAC-CPF, nous avons ainsi réfléchi à une solution permettant de créer une sortie compatible avec ce schéma sans perte d'information ${ }^{27}$. L'extension ExportEacCpf, construite

27. Le site officiel d'Omeka indique les points principaux à suivre pour créer une nouvelle sortie en fonction des versions du système et les développeurs de l'équipe Omeka 
selon le modèle de l'extension METS Export, dont la structure et les finalités s'apparentent aux nôtres, est disponible dans un entrepôt GitHub ${ }^{28}$. Elle permet d'ajouter le format EAC-CPF aux formats de sortie, situés à la fin de chaque fiche d'autorité. La création de cette extension nous a ainsi permis de boucler le flux des données en obtenant en sortie encore un fichier qui, avec quelques menus réaménagements, peut être validé contre un schéma EAC-CPF.

\section{La publication de la base en ligne}

Le site, qui constitue l'aboutissement du projet, http://josticeetplet. huma-num.fr, est hébergé par Huma-Num à travers le service NAKALONA, une instance du logiciel Omeka en mode SAAS. Des liens ont également été mis en place à partir de l'édition du Livre de jostice et de plet (Élec) vers la base prosopographique.

Les contenus du site sont organisés dans deux collections :

- «Personne »: collection contenant les notices des personnes mentionnées dans le Livre de jostice et de plet;

- «Bibliographie » : collection contenant les références d'ouvrages ou d'autres documents comportant des informations sur les personnes citées dans le Livre de jostice et de plet.

À ce stade, la collection «Personne » ne comporte que les personnes explicitement citées dans la contribution de Henri Stein. Par contre, la collection «Bibliographie » intègre aux ressources indiquées par H. Stein dans le cadre de ses recherches d'autres ressources évoquées dans le projet, sans toutefois être assimilable à une bibliographie complète concernant le Livre de jostice et de plet. Les éléments de la collection «Bibliographie » ont été importés depuis la base bibliographique créée avec Zotero, à travers l'extension Zotero Import mentionnée plus haut, sans mise en forme graphique.

La solution décrite permet de gérer la chaîne éditoriale des notices d'autorité, de leur création jusqu'à la visualisation et diffusion en ligne et au téléchargement des fichiers. En tant que modèle mis en place dans des délais raisonnablement courts, il peut sans doute donner lieu à des améliorations et à des développements plus ou moins approfondis et élaborés en fonction des exigences du projet envisagé, qui devront forcément prendre en compte la mise à jour du système et des extensions utilisées. La structuration des fiches EAC-CPF/XML, qui comporte actuellement des éléments englobants

sont particulièrement réactifs sur le forum Omeka. Des indications de base pour la création d'un nouveau format d'exportation sont disponibles ici : <https://omeka.org/codex/Plugin_ Use_Cases/Add_a_New_Output_Format $>$ ainsi que dans nos échanges avec les développeurs $<$ https://forum.omeka.org/t/add-a-new-xml-output-format/296>.

28. <https://github.com/sgraziella/ExportEacCpf>. 


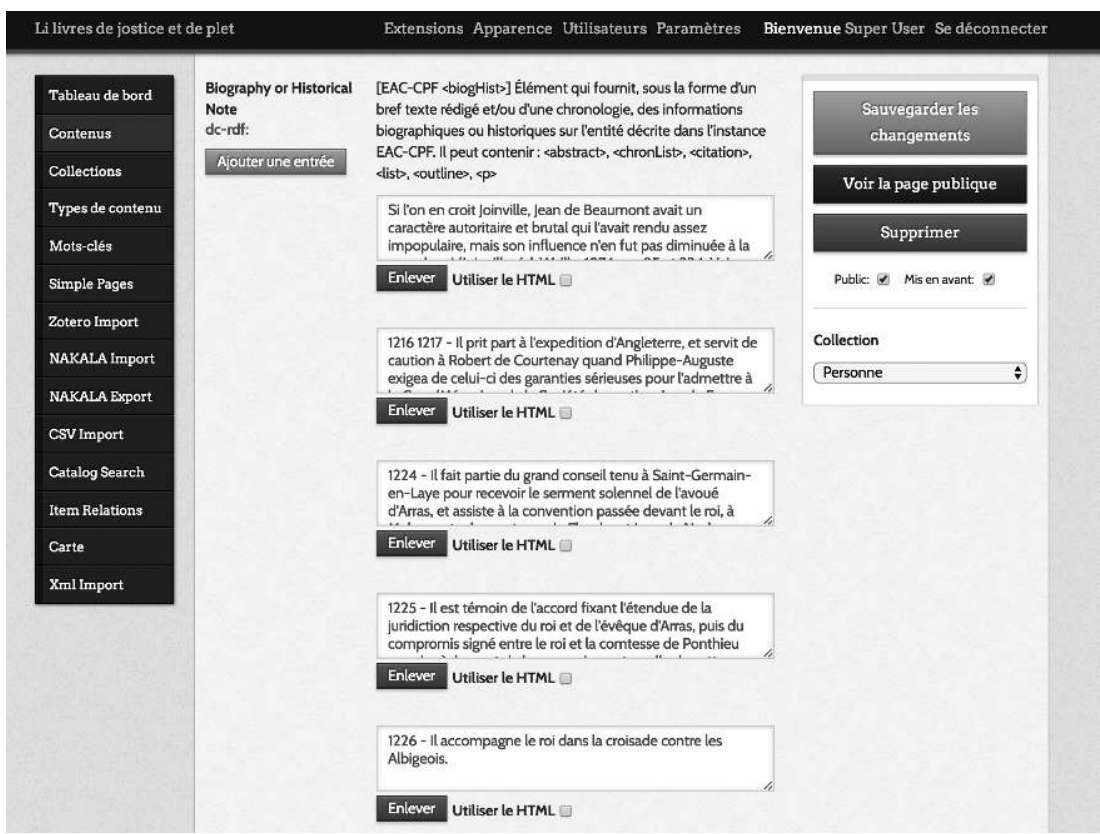

Source : Interface d'administration du CMS Omeka.

Fig. 6. L'élément <biogHist> importé dans Omeka

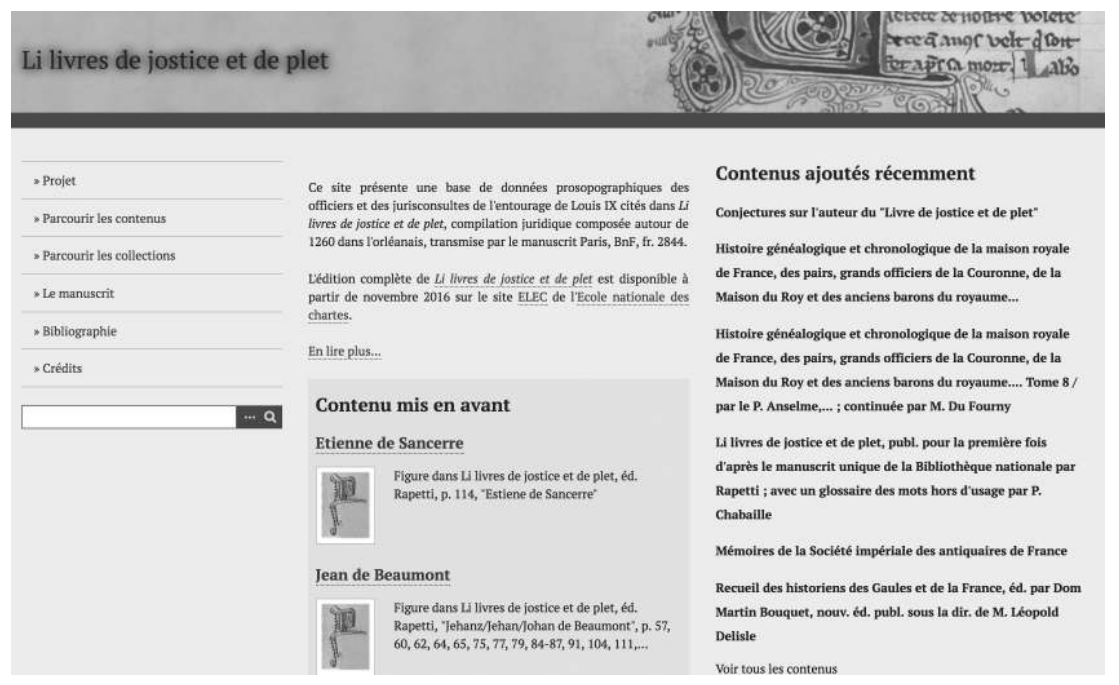

Source : Interface d'administration du CMS Omeka.

7. Page d'accueil du site <http://josticeetplet.huma-num.fr/> 
dont la structure interne doit être partiellement rétablie dans le format de sortie, pourra notamment être peaufinée en retravaillant la feuille de style XSLT qui permet l'importation des fichiers XML (XML Import) ; une autre piste d'amélioration consiste à fournir des règles typographiques pour les éléments englobants, par exemple introduisant des éléments de ponctuation pour différencier les éléments internes afin d'appliquer des expressions régulières en sortie.

Ce modèle de travail, préconisant un système de publication et de gestion de fichiers d'autorité, a été l'occasion de réfléchir de manière plus approfondie au milieu de production du Livre de jostice et de plet et de faire apparaître de manière plus évidente les relations entre les personnes évoquées par le compilateur. Des développements techniques, tels que la visualisation des données sur une frise chronologique ${ }^{29}$ ou l'intégration d'extraits de l'édition du texte ${ }^{30}$, ainsi que l'élargissement du corpus, pourront intervenir dans une deuxième phase de ce projet.

Graziella Pastore - École nationale des chartes (Centre Jean Mabillon), Labex Hastec, PSL Research University (Paris Sciences \& Lettres)

29. Par exemple à travers l'extension Neatline <http://neatline.org/>.

30. Par exemple à travers l'extension Bookmeka <http://bookmeka.huma-num.fr/>. 
L'édition numérique du Livre de jostice et de plet (Élec), point de départ de la constitution d'une base prosopographique (Omeka)

La solution décrite dans cette contribution permet de combiner exigences académiques et difficultés pratiques : un outil de gestion de fichiers qui permet de gérer des fichiers d'autorité, d'en visualiser les données comme de s'assurer de leur interopérabilité, sans pour autant nécessiter une formation ardue et tout en s'adaptant à des corpus et des projets variés. Les fichiers d'autorité ont été balisés en XML en suivant le format EAC-CPF (Encoded Archival Context - Corporate Bodies, Persons and Families) ; la visualisation des données est gérée par le système de gestion de contenus (CMS) Omeka. Le corpus d'autorité est basé sur les officiers et jurisconsultes cités dans le Livre de jostice et de plet, compilation juridique rédigée en ancien français au XIII ${ }^{e}$ siècle et récemment éditée en ligne sur Élec (Éditions en ligne de l'École des chartes).

Édition numérique - humaniés numériques - Livre de jostice et de plet prosopographie - Omeka.

The Scholarly Digital Edition of the Livre de jostice et de plet (Élec): A Starting Point for Creating a Prosopographical Database with Omeka CMS

The solution described is the result of a combination of research and practical experience: a file management tool for authority files, with publishing and interoperable capabilities that can be handled without a steep learning curve, and that can adapt themselves to variation or different cases. The authority records were delineated in XML markup following EAC-CPF (Encoded Archival Context-Corporate Bodies, Persons and Families) convention; the dataflow was managed by the Omeka Content Management System. The corpus of authority files is based on officials and jurisconsults cited in the Livre de jostice et de plet, a legal compilation written in Old French around 1260, recently edited online on Élec (Éditions en ligne de l'École des chartes). Digital Edition - Digital Humanities - Livre de jostice et de plet Prosopography - Omeka. 
